\title{
Superconductivity and Cooper Pairs
}

\author{
Mitramani Subedi \\ Department of Physics Prithvi Narayan Campus, Pokhara \\ Email: subedimitramani@gmail.com
}

\begin{abstract}
In this article, some crucial information is given in regard to superconductivity. The advancement in high temperature superconductivity is believed to solve the problem of energy crisis. So, intensive research in this part of physics is recommended. Actually, Cooper pairs of electrons are responsible for superconductivity.

Key words: Critical temperature, persistent current, Cooper pair, phonon interaction
\end{abstract}

\section{INTRODUCTION}

In condensed matter physics, a Cooper pair is a pair of electrons (or other fermions) bound together at low temperatures in a certain manner first described in 1956 by American physicist Cooper. Cooper showed that an arbitrarily small attraction between electrons in a metal can cause a paired state of electrons to have a lower energy than the Fermi energy, which implies that the pair is bound. In conventional superconductors, this attraction is due to the electro-phonon interaction. The Cooper pair state is responsible for superconductivity, as described in the BCS theory developed by John Bardeen, Leon Cooper, and John Schrieffer for which they shared the 1972 Nobel Prize [3]. Although Cooper pairing is a quantum effect, the reason for the pairing can be seen from a simplified classical explanation. An electron in a metal normally behaves as a free particle. The electron is repelled from other electrons due to their negative charge, but it also attracts the positive ions that make up the rigid lattice of the metal. This attraction distorts the ion lattice, moving the ions slightly toward the electron, increasing the positive charge density of the lattice in the vicinity. This positive charge can attract other electrons. At long distances, this attraction between electrons due to the displaced ions can overcome the electrons' repulsion due to their negative charge, and cause them to pair up. The rigorous quantum mechanical explanation shows that the effect is due to electron-phonon interactions, with the phonon being the collective motion of the positively-charged lattice [5].

The energy of the pairing interaction is quite weak, of the order of $10^{-3} \mathrm{eV}$, and thermal energy can easily break the pairs. So only at low temperatures, in metal and other substrates, are a significant number of the electrons in Cooper pairs.

The electrons in a pair are not necessarily close together; because the interaction is long range, paired electrons may still be many hundreds of nanometers apart. This distance is usually greater than the average inter-electron distance; so many Cooper pairs can occupy the same space. Electrons have spin- $-\frac{1}{2}$, so they are fermions, but the total spin of a Cooper pair is integer ( 0 or 1$)$ so it is a composite boson. This means the wave functions are symmetric under particle interchange. Therefore unlike electrons, multiple Cooper pairs are allowed to be in the same quantum state.

The BCS theory is also applicable to other fermions systems, such as helium-3. Indeed, Cooper pairing is responsible for the superfludity of helium-3 at low temperatures. It has also been recently demonstrated that a Cooper pair can comprise two bosons. Here, the pairing is supported by entanglement in an optical lattice.

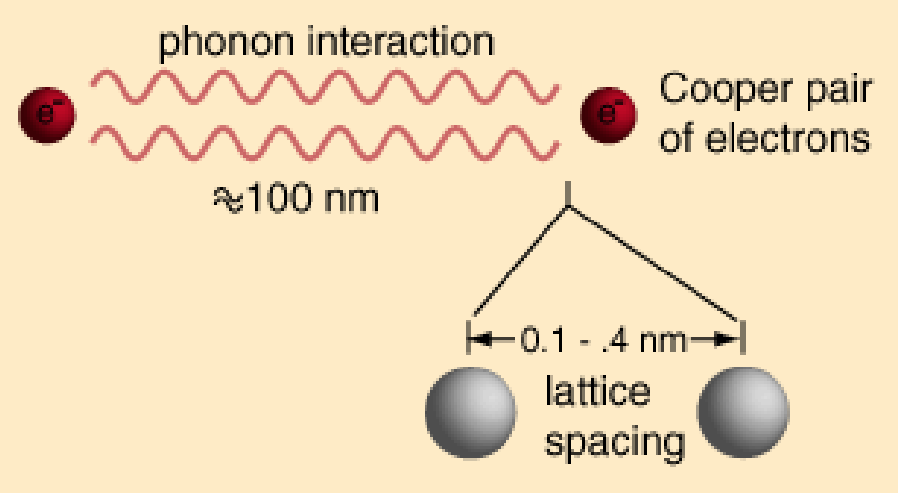

Fig. 1 Cooper pairs

The behavior of superconductors suggests that electron pairs are coupling over a range of hundreds of nanometers, three orders of magnitude larger than the lattice spacing called Cooper pairs, these coupled electrons can take the character of a boson and condense into the ground state. This pair condensation is the basis for the BCS theory of superconductivity. The effective net attraction between the normally repulsive electrons produces a pair binding energy on the order of milli-electron volts, enough to keep them 
paired at extremely low temperatures.

The transition of a metal from the normal to the superconducting state has the nature of a condensation of the electrons into a state which leaves a band gap above them. This kind of condensation is seen with superfluid helium, but helium is made up of bosons -- multiple electrons can't collect into a single state because of the Pauli Exclusion Principle. Froehlich was first to suggest that the electrons act as pairs coupled by lattice vibrations in the material. This coupling is viewed as an exchange of phonons, phonons being the quanta of lattice vibration energy. Experimental corroboration of an interaction with the lattice was provided by the isotope effect on the superconducting transition temperature. The boson-like behavior of such electron pairs was further investigated by Cooper and they are called "Cooper pairs". The condensation of Cooper pairs is the foundation of the BCS theory of superconductivity.

If a superconductor has the form of a ring and current is once established in it by some means, the current, even several hundred amperes, flows indefinitely, even for over a year, without any change in its value. Such currents are called persistent currents [2].

\section{A MODEL OF COOPER PAIR ATTRACTION}

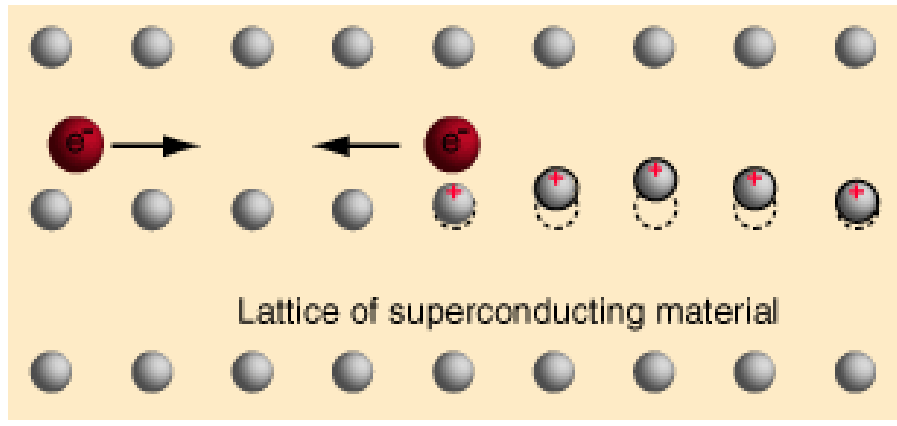

A passing electron attracts the lattice, causing a slight ripple toward its path.

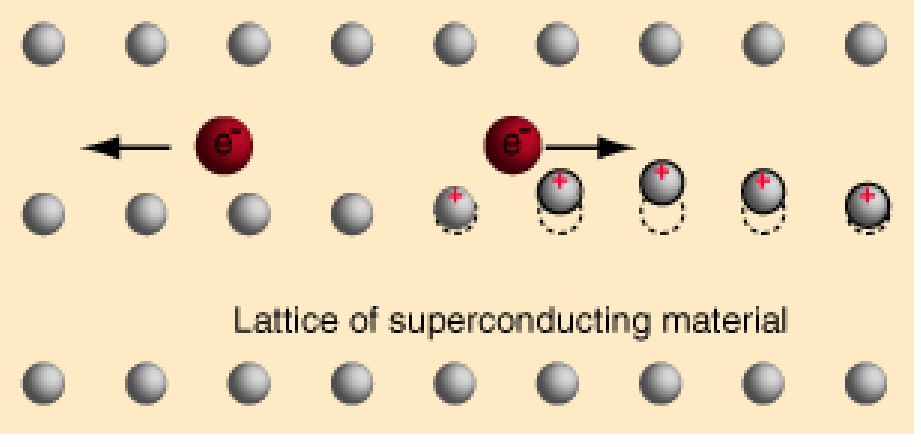

Fig. 2

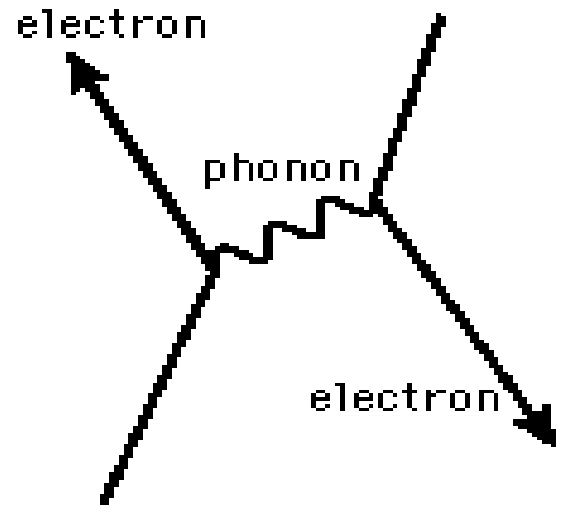

Fig. 3

Another electron passing in the opposite direction is attracted to that displacement.

A visual model of the Cooper pair attraction has a passing electron which attracts the lattice,causing a slight ripple toward its path[1].Another electron passing in the opposite direction is attracted to that displacement. This constitutes a coupling between electrons which can be depicted in a Feynman diagram. As strange as such an interaction seems, it is experimentally supported by the isotope effect and the evidence for a condensation at the critical temperature for superconductivity.

\section{ISOTOPE EFFECT, MERCURY}

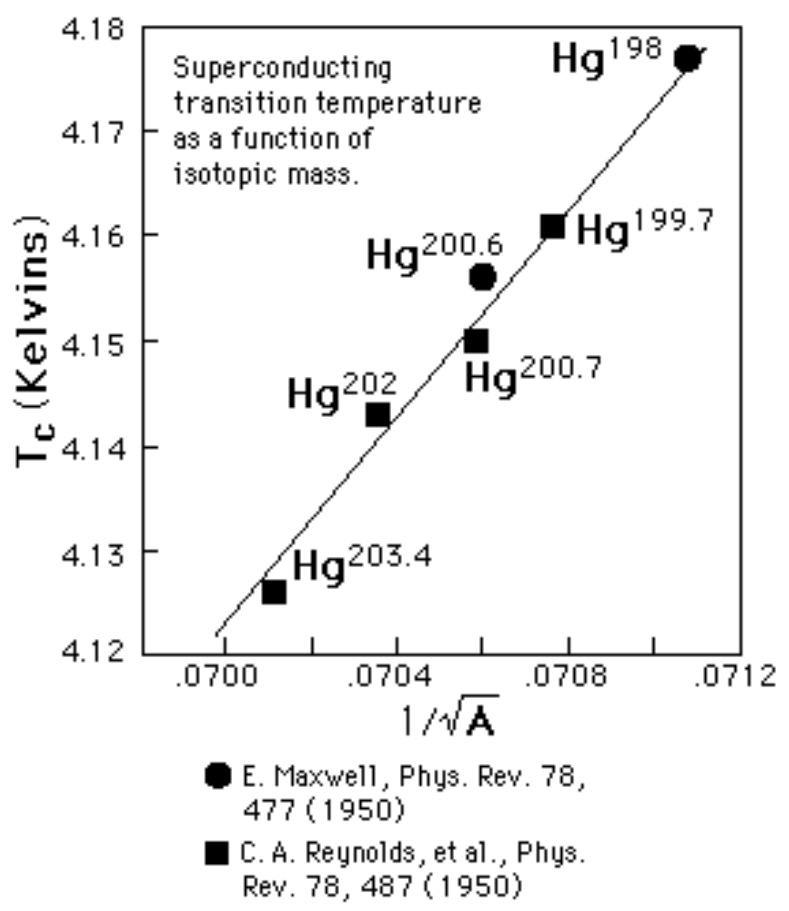

Fig. 3

If electrical conduction in mercury were purely electronic, there should be no dependence upon the nuclear masses. This 
dependence of the critical temperature for superconductivity upon isotopic mass was the first direct evidence for interaction between the electrons and the lattice. This supported the BCS theory of lattice coupling of electron pairs.

It is quite remarkable that an electrical phenomenon like the transition to zero resistivity should involve a purely mechanical property of the lattice [4]. Since a change in the critical temperature involves a change in the energy environment associated with the superconducting transition, this suggests that part of the energy is being used to move the atoms of the lattice since the energy depends upon the mass of the lattice. This indicates that lattice vibrations are a part of the superconducting process. This was an important clue in the process of developing the BCS theory because it suggested lattice coupling, and in the quantum treatment suggested that phonons were involved.

\section{MEASURED SUPERCONDUCTOR BAND- GAP}

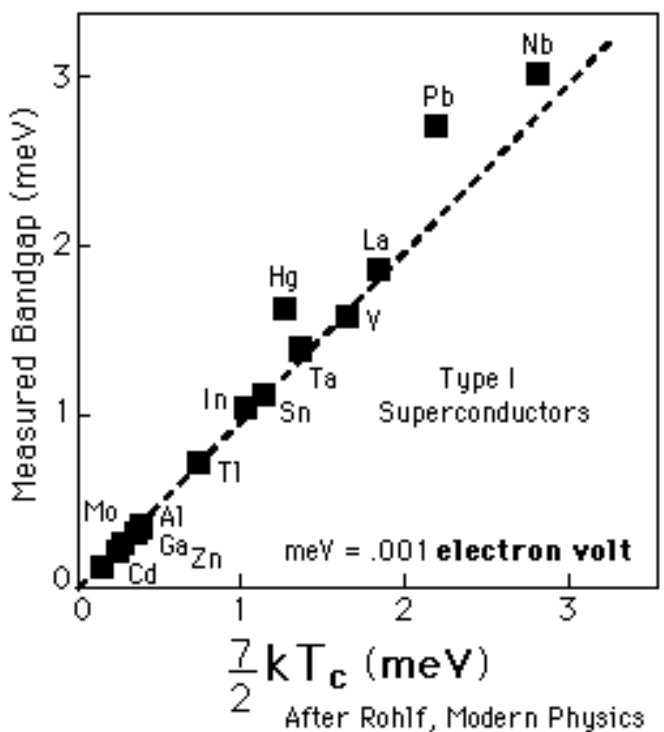

Fig. 5

The measured band gap in Type I superconductors is one of the pieces of experimental evidence which supports the BCS theory. The BCS theory predicts a band gap of

$$
\mathrm{E}_{\mathbf{g}} \approx \frac{7}{2} \mathrm{k} \mathrm{T}_{\mathbf{c}}
$$

where $T_{c}$ is the critical temperature for the superconductor. The energy gap is related to the coherence length for the superconductor, one of the two characteristic lengths associated with superconductivity.

\section{ENERGY GAP IN SUPERCONDUCTORS AS A FUNCTION OF TEMPERATURE}

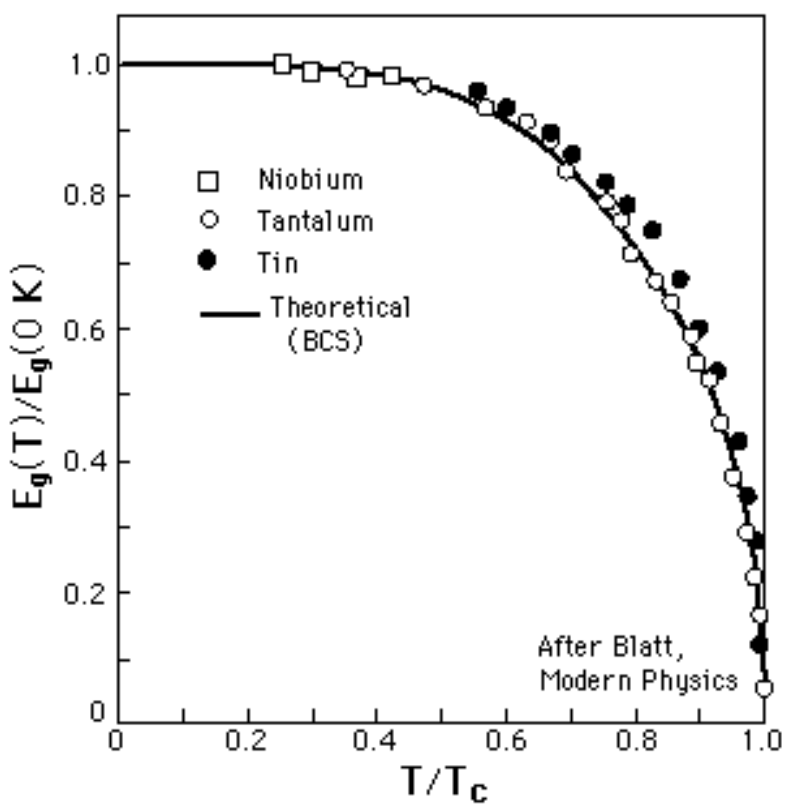

Fig. 6

The effective energy gap in superconductors can be measured in microwave absorption experiments. The data at left offer general confirmation of the BCS theory of superconductivity. The data is attributed to Townsend and Sutton.

The reduction of the energy gap as you approach the critical temperature can be taken as an indication that the charge carriers have some sort of collective nature. That is, the charge carriers must consist of at least two things which are bound together, and the binding energy is weakening as you approach the critical temperature. Above the critical temperature, such collections do not exist, and normal resistivity prevails [3]. This kind of evidence, along with the isotope effect which showed that the crystal lattice was involved, helped to suggest the picture of paired electrons bound together by phonon interactions with the lattice.

\section{CONCLUSION}

The Cooper pairs of electrons have a peculiar property of smoothly sailing over the lattice points without any energy exchange i.e. the cooper pairs are not scattered by the lattice points. Hence no transfer of energy takes place from the electron pair to the lattice ions. If an electric 
field is established inside the substance, the electrons gain additional kinetic energy and give rise to a current. But they do not transfer this energy to the lattice, so that they do not get slowed down. As a consequence of this, the substance does not possess any electrical resistivity. In fact, the tendency for all the Cooper pairs in a body to condense into the same ground quantum state is responsible for the peculiar properties of superconductivity.

\section{REFERENCES}

1. Cooper pair-Wikipedia

2. Murugeshan R.;Sivaprasath Kiruthiga,Fifth Edition(1995) (S.CHAND \& COMPANY LTD. RAMANAGAR, NEW DELHI-110055) p. 416-418, ISBN:81-219-0210-X.

3 Cooper,Leon N. (1956)."Bound electron pairs in a degenerate Fermi gas".Physical Review,104 (4):11891190 .

4. Feynman,Richard P.:Leighton,Robert:Sands,Ma thew(1965). Lectures on Physics,Vol.3 AddisonWeslev.p.21-;ISBN 0-201-02118-8.

5. J.Barden,"Electron-Phonon Interactions and Superconductivity",in Cooperative Phenomena (Springer-Verlag,Berlin,Heidelberg,New York,1973) ,p.67. 